\title{
Randomized Trial of Intravenous Versus Intraperitoneal Chemotherapy Plus Bevacizumab in Advanced Ovarian Carcinoma: An NRG Oncology/Gynecologic Oncology Group Study
}

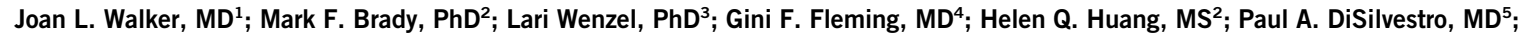

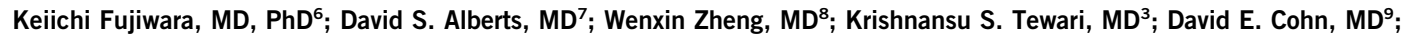
Matthew A. Powell, MD ${ }^{10}$; Linda Van Le, MD ${ }^{11}$; Susan A. Davidson, MD ${ }^{12}$; Heidi J. Gray, MD ${ }^{13}$; Peter G. Rose, MD ${ }^{14}$; Carol Aghajanian, MD ${ }^{15}$; Tashanna Myers, MD ${ }^{16}$; Angeles Alvarez Secord, MD ${ }^{17}$; Stephen C. Rubin, MD ${ }^{18}$; and Robert S. Mannel, MD

ASSOCIATED CONTENT

See accompanying Oncology Grand Rounds on page 1359 Data Supplements Author affiliations and support information (if applicable) appear at the end of this article.

Accepted on February 4, 2019 and published at jco.org on April 19, 2019: DOI https://doi.org/10. $1200 / J C 0.18 .01568$

Clinical trial information: NCT00951496.

PURPOSE To evaluate the impact of two different intraperitoneal (IP) chemotherapy regimens on progressionfree survival (PFS) among women with newly diagnosed advanced ovarian carcinoma.

METHODS Eligible patients were randomly assigned to six cycles of IV paclitaxel $80 \mathrm{mg} / \mathrm{m}^{2}$ once per week with intravenous (IV) carboplatin area under the curve 6 (IV carboplatin) versus IV paclitaxel $80 \mathrm{mg} / \mathrm{m}^{2}$ once per week with IP carboplatin area under the curve 6 (IP carboplatin) versus once every 3 weeks IV paclitaxel $135 \mathrm{mg} / \mathrm{m}^{2}$ over 3 hours day 1 , IP cisplatin $75 \mathrm{mg} / \mathrm{m}^{2}$ day 2 , and IP paclitaxel $60 \mathrm{mg} / \mathrm{m}^{2}$ day 8 (IP cisplatin). All participants received bevacizumab $15 \mathrm{mg} / \mathrm{kg}$ IV every 3 weeks in cycles 2 to 22 .

RESULTS A total of 1,560 participants were enrolled and had 84.8 months of follow-up. The median PFS duration was 24.9 months in the IV carboplatin arm, 27.4 months in the IP carboplatin arm, and 26.2 months in the IP cisplatin arm. For the subgroup of 1,380 patients with stage II/III and residual disease of $1 \mathrm{~cm}$ or less, median PFS was 26.9 (IV-carboplatin), 28.7 (IP-carboplatin), and 27.8 months (IP cisplatin), respectively. Median PFS for patients with stage II/III and no residual disease was 35.9, 38.8, and 35.5 months, respectively. Median overall survival for all enrolled was 75.5, 78.9, and 72.9 months, respectively, and median overall survival for stage II/III with no gross residual disease was 98.8 months, 104.8 months, and not reached. Mean patient-reported Functional Assessment of Cancer Therapy neurotoxicity scores (Gynecologic Oncology Group) were similar for all arms, but the mean Trial Outcome Index of the Functional Assessment of Cancer Therapy-Ovary scores during chemotherapy were statistically worse in the IP cisplatin arm.

CONCLUSION Compared with the IV carboplatin reference arm, the duration of PFS was not significantly increased with either IP regimen when combined with bevacizumab and was better tolerated than IP cisplatin.

J Clin Oncol 37:1380-1390. ๑ 2019 by American Society of Clinical Oncology

\section{INTRODUCTION}

Ovarian cancer incidence in 2018 was expected to be 22,240 with 14,070 deaths. ${ }^{1}$ This study was designed to build on the advances seen with intraperitoneal (IP) cisplatin and paclitaxel administration as demonstrated by the Gynecologic Oncology Group (GOG) protocol 172, where women with stage III disease and completely resected disease survived a median of 127.6 months with IP chemotherapy compared with 82 months with intravenous (IV) cisplatin-based chemotherapy. ${ }^{2-4}$ Despite the survival benefit, less than half of eligible women treated at National Cancer Institute ( $\mathrm{NCl}$ ) comprehensive cancer centers received this treatment secondary to toxicity and the difficulty with administering IP therapy as reported by Wright et al. ${ }^{5}$ The ovarian committee of the GOG determined that a less complicated, less toxic, more feasible outpatient regimen was needed to increase access. The performance of phase I studies GOG9916, GOG-9917, and GOG-9921 $1^{6-8}$ helped to identify tolerable IP chemotherapy regimens.

The community standard of carboplatin and paclitaxel IV every 3 weeks was challenged by improved survival demonstrated with weekly paclitaxel and every 3 weeks carboplatin in the Japanese Gynecologic Oncology Group (JGOG) study 3016. This led to selection of weekly paclitaxel and every 3 weeks carboplatin as the control arm for the current study 
(GOG-252). ${ }^{9,10}$ In addition, a separate clinical trial, GOG$262,{ }^{11}$ was designed to confirm the JGOG results in the US population. Immediately before activation of GOG-252 and GOG-262, the activity of bevacizumab in this patient population was becoming evident. There was concern that withholding bevacizumab would decrease enrollment and adherence to the protocol and result in a biased survival analysis because the availability of bevacizumab postrecurrence may alter results. ${ }^{12-14}$ The preliminary results from GOG-218 and the International Collaborative Ovarian Neoplasm (ICON) trial ICON-7, which evaluated the addition of bevacizumab in primary therapy of ovarian cancer, were completed at approximately the same time of opening GOG-252. ${ }^{15,16}$ Therefore, bevacizumab was added to all arms of the current trial because it was assumed that including bevacizumab in each study regimen would not appreciably alter the relative effectiveness of the chemotherapy regimens.

\section{METHODS}

Eligible patients with newly diagnosed stage II through IV epithelial ovarian, fallopian tube, or primary peritoneal cancer were enrolled within 12 weeks of surgery for staging and maximal cytoreduction. A GOG performance status score of 0 to 2 was required. Eligibility included adequate laboratory assessment, including creatinine levels no higher than the upper limit of normal, and patients could not have any contraindications to bevacizumab (see the Data Supplement for full eligibility criteria). All patients provided written institutional review board-approved informed consent.

\section{Study Design}

GOG-252 is an open-label, randomized phase III clinical trial to determine whether IP chemotherapy would improve progression-free survival (PFS) compared with the reference IV arm paclitaxel $80 \mathrm{mg} / \mathrm{m}^{2}$ once per week with IV carboplatin area under the curve (AUC) 6 . The study was originally designed for patients with stage II/III optimally resected disease. An exploratory objective was added to include patients with suboptimal stage III and stage IV disease. The experimental IP arms were IV paclitaxel $80 \mathrm{mg} / \mathrm{m}^{2}$ once per week with IP carboplatin AUC 6 (IP carboplatin) and IV paclitaxel $135 \mathrm{mg} / \mathrm{m}^{2}$ over 3 hours day 1 , day 2 IP cisplatin $75 \mathrm{mg} / \mathrm{m}^{2}$, and day 8 IP paclitaxel $60 \mathrm{mg} / \mathrm{m}^{2}$ (IP cisplatin). All arms delivered bevacizumab $15 \mathrm{mg} / \mathrm{kg}$ IV day 1 cycles 2 to 22 (NSC \#704865, IND \#7921; Fig 1). Secondary objectives were improvement in overall survival (OS), toxicity of each arm, and patientreported outcomes (PROs) to determine quality of life while on each regimen.

\section{Disease Assessment}

Computed tomography scans or magnetic resonance imaging of the abdomen and pelvis was obtained for documentation of measurable disease but was not the basis for stratification or eligibility. Imaging (computed tomography scan or magnetic resonance imaging), cancer antigen 125 measurement, and physical examination were to be repeated after treatment cycles 6,12 , and 22 of chemotherapy and every 6 months until 5 years from enrollment and then annually until progression or death. Toxicity monitoring and dose adjustments are provided in the Data Supplement.

\section{Quality-of-Life Assessments and Instruments}

PROs were assessed at six time points: before random assignment, before the fourth cycle ( 9 weeks after starting treatment), before the seventh cycle (18 weeks after starting treatment), before the 13th cycle (36 weeks after starting treatment), before the 21st cycle (60 weeks after starting treatment), and 84 weeks after starting treatment. The Trial Outcome Index of the Functional Assessment of Cancer Therapy-Ovary (FACT-O TOI) was used to capture patient-reported assessments of their own physical and functional well-being and additional concerns related specifically to ovarian cancer. ${ }^{14,17,18}$ Lower FACT-O TOI scores represent poorer quality of life. In addition, the fouritem FACT/GOG-Neurotoxicity subscale (short), the fouritem FACT/GOG-Abdominal Discomfort subscale, the 13-item Functional Assessment of Chronic Illness TherapyFatigue subscale, and a single-item nausea question were used to capture the patient's self-reported chemotherapyinduced neuropathy, abdominal discomfort, and fatigue, respectively. All measures were scored using a 5-point scale (not at all $=0$, a little bit $=1$, somewhat $=2$, quite a bit $=3$, very much $=4$ ).

\section{Statistical Considerations}

Study treatment was assigned using a minimization procedure. ${ }^{19}$ This procedure randomly allocated one of the three study treatments such that there would be nearly an equal number of patients who received each study treatment within the following patient-level characteristics: cancer stage and residual disease status (II $v$ III with no gross residual $v$ III with gross residual $v$ IV). The primary study end point was PFS calculated from each patient's enrollment date to the onset of either clinically evident progression or death as a result of any cause, whichever occurred first. The onset of clinical progression was defined as either radiographic evidence of increasing disease on the basis of Response Evaluation Criteria in Solid Tumors (RECIST) version 1.1, a global deterioration of health, or a rise in cancer antigen 125 level using the Gynecologic Cancer Intergroup criteria. ${ }^{20,21}$ The duration of PFS was censored at the date of last contact for those patients who remained alive and free of progression. ${ }^{19}$ The trial was designed to establish the superiority of each of the IP regimens compared with the IV regimen using a stratified log-rank test. ${ }^{22}$ The two IP regimens are compared with each other if superiority is found. The null hypotheses (ie, equivalence of the hazard of progression or death between 


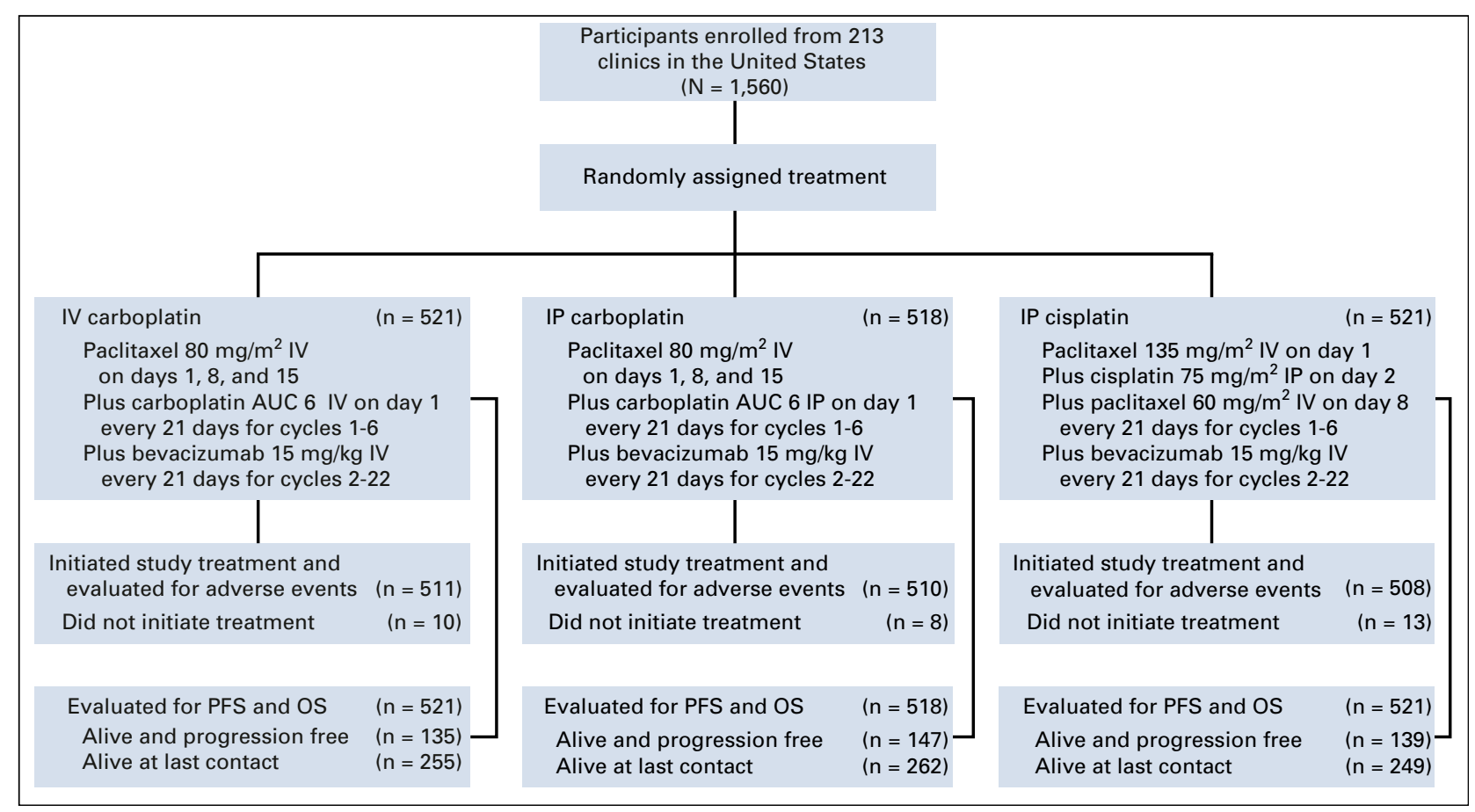

FIG 1. CONSORT diagram that accounts for all participants and the chemotherapy regimen arms to which they were randomly assigned. Crossover during treatment to the IV arm occurred in $16 \%$ of those randomly assigned to the IP carboplatin arm and $28 \%$ of those randomly assigned to the IP cisplatin arm. Taxotere was substituted for paclitaxel in 7\% of those in the IV and IP carboplatin arms and $5 \%$ of those in the IP cisplatin arm. There was discontinuation of bevacizumab during the concurrent chemotherapy administration of the first six cycles in $15 \%$ of participants in the IV and IP carboplatin arms and $30 \%$ of participants in the IP cisplatin arm. The median number of cycles of bevacizumab was 20 for those in the IV carboplatin arm, 19 in the IP carboplatin arm, and 17 in the IP cisplatin arm. Reason for discontinuation of study regimen was completion of therapy in $51 \%$ of participants in the IV carboplatin arm, $49 \%$ in the IP carboplatin arm, and $45 \%$ in the IP cisplatin arm. Toxicity caused discontinuation in $24 \%, 28 \%$, and $29 \%$, respectively, and refusal was $6 \%, 6 \%$, and $9 \%$, respectively. Disease progression or death was cause for discontinuation in 16\%, 13\%, and 13\%, respectively. The delivery of six cycles of any platinum agent was $90 \%$ in the IV and IP carboplatin arms and $84 \%$ in the IP cisplatin arm. Day 8 paclitaxel was delivered to $66 \%$ of participants in the IV or IP carboplatin arms for all six cycles and only $59 \%$ in the IP cisplatin arm. Four cycles of day 8 paclitaxel was received by $85 \%$ of participants in the IV and IP carboplatin arms and $80 \%$ of those in the IP cisplatin arm. In the IV and IP carboplatin arms, only $40 \%$ of participants received day 15 paclitaxel through six cycles, and $60 \%$ received it through four cycles, whereas $80 \%$ received two cycles. AUC, area under the curve; OS, overall survival; PFS, progression-free survival.

each of the two IP regimens compared with the IV regimen) was assessed with a stratified log-rank test. ${ }^{22}$ The primary analyses of PFS included all patients enrolled in the study regardless of eligibility or compliance with their assigned study regimen intention to treat. The design also allowed for comparing treatments within the subgroup of individuals with International Federation of Gynecology and Obstetrics stage II or III disease and no residual disease greater than 1 $\mathrm{cm}$. The overall type I error for these hypotheses was to be limited to 5\% (one tail), after accounting for the correlation between hypothesis tests because of a common reference group (IV regimen), and testing within a subgroup (stage II/III optimally resected). The study was considered sufficiently mature for a final analysis when at least 360 first progressions or death (PFS events) had occurred among those allocated to the reference regimen IV carboplatin. This sample size provides approximately $80 \%$ power for detecting a $20 \%$ decrease in the hazard of first progression or death when each IP regimen is compared with the IV regimen in the intention-to-treat population (Data Supplement). $\mathrm{NCl}$ Common Terminology Criteria for Adverse Events (version 3.0) was used to grade the adverse events. ${ }^{23}$ Summaries and analyses of adverse events include only those patients who at least initiated their assigned study treatment.

For the analysis of PROs, a mixed-effects model was used to compare the mean PRO scores between treatment groups (see Data Supplement for a description of all measures). If there was evidence of a time-dependent treatment effect, Hochberg step-down procedure was used to control the type I error of between-treatment comparisons over the individual assessment times. ${ }^{24}$ The least squares mean estimates were obtained from a fitted mixed-effects model that adjusted for pretreatment score (baseline score) and patient age at the time of enrollment. The treatment differences were estimated from the fitted 
mixed-effects models and presented with the corresponding 99\% Cls. The effect of study treatments on nausea was summarized with patients' complaints of somewhat nauseous or having worse nausea and was evaluated with the generalized estimating equation method that adjusted for baseline score and patient age. To control the overall type I error to be 5\% for the entire family of PRO hypotheses, a significance level for each of the five PRO measures was set to $1 \%$ (two sided) for the global treatment comparison. As a result, the reported $P$ values were Bonferroni adjusted. Compliance across regimens was assessed with methods using generalized estimating equations.

\section{RESULTS}

This report includes an accounting of all patients who were enrolled and randomly assigned. In total, 1,560 patients with epithelial ovarian, fallopian tube, and peritoneal carcinoma were enrolled between July 27, 2009, and November 30, 2011. The study was designed to determine whether IP chemotherapy improves PFS in optimally resected $(\leq 1 \mathrm{~cm})$ advanced-stage epithelial ovarian, fallopian tube, and peritoneal carcinoma (Fig 1). The database was frozen and locked for this analysis on November 27, 2018, and there have been 84.8 months of follow-up.

Participants were a median age of 58 years. Seventy-two percent had primary tumors with a high-grade serous histology, 58\% had only microscopic residual disease (no gross residual by surgical report), 93\% had residual tumor of $1 \mathrm{~cm}$ or less, and $84 \%$ had stage III disease (Table 1).

\section{Efficacy}

At the time of these analyses, 1,139 of all the enrolled patients (73\%) experienced disease progression or death. The median duration of PFS was 24.9 months for patients randomly assigned to IV carboplatin, 27.4 months for those assigned to IP carboplatin, and 26.2 months for those assigned to IP cisplatin (Fig 2). Compared with IV carboplatin, the hazard of first progression or death was $7.5 \%$ lower (hazard ratio [HR], $0.925 ; 95 \% \mathrm{Cl}, 0.802$ to 1.07 ) for the IP carboplatin arm and $2.3 \%$ lower (HR, $0.977 ; 95 \% \mathrm{Cl}$ 0.847 to 1.13 ) for the IP cisplatin arm. There was no statistically significant difference in PFS between the IV regimen and either of the IP regimens.

Among the 1,380 participants who had stage II and III and optimally resected to $1 \mathrm{~cm}$ or less residual disease per surgeon, $973(71 \%)$ experienced progression or death. In this subgroup of patients, the median duration of PFS was 26.9 months for IV carboplatin, 28.7 months for IP carboplatin (HR, $0.921 ; 95 \% \mathrm{Cl}, 0.789$ to 1.07 ), and 27.8 months for IP cisplatin (HR, 0.966; 95\% Cl, 0.828 to 1.13). There was no statistically significant difference in PFS between the IV regimen and either of the IP regimens in this subgroup of patients (Fig 3 ).
There were 870 participants with stage II or III and no gross residual disease documented at the completion of surgery, of whom 534 (61\%) experienced disease progression or death. In this subgroup of patients, the median duration of PFS was 35.9 months for IV carboplatin, 38.8 months for IP carboplatin, and 36.5 months for IP cisplatin. There was no statistically significant difference in PFS between the IV regimen and either of the IP regimens in this subgroup of patients (Fig 4).

For all randomly assigned participants, with $49 \%$ alive after a median follow-up of 84.8 months, the median OS was 75.5 months in the IV carboplatin arm and 78.9 and 72.9 months for the IP carboplatin and IP cisplatin arms, respectively. Relative to the IV carboplatin group, the hazard of death was similar in the IP carboplatin arm (HR, $0.949 ; 95 \% \mathrm{Cl}, 0.799$ to 1.128 ) and IP cisplatin arm (HR, 1.05 ; $95 \% \mathrm{Cl}, 0.884$ to 1.24; Fig 5). OS for stage III optimally resected to $1 \mathrm{~cm}$ or less residual disease was 74.6, 78.2, and 74.1 months, respectively (Data Supplement). For stage II/III optimally resected with $1 \mathrm{~cm}$ or less residual disease, the OS was 80.0, 84.7, and 76.3 months, respectively (Data Supplement). For stage II/III with no gross residual disease, the median OS was 98.8 months for IV carboplatin, 104.8 months for IP carboplatin, and not reached for IP cisplatin (Data Supplement).

\section{Safety}

Twenty-five deaths were attributed, in part, to toxicity (eight IV carboplatin, seven IP carboplatin, 10 IP cisplatin), five of which were during cycle 1 , eight during cycles 2 to 4 , seven during cycles 5 to 6 , and five after completing cycle 6 . Three deaths were cardiac, one CNS, four sudden death not otherwise characterized, seven associated with sepsis during the first six cycles, two associated with Gl perforations, and the remaining deaths as a result of disease progression or an unspecified cause (Table 2).

Grade 3 or worse infections were higher ( $P=.008$ global exact test) in the IP arms (11.5\% for IV carboplatin, 17.2\% for IP carboplatin, and $17.7 \%$ for IP cisplatin). Growth factors were added, and day 15 paclitaxel was discontinued in $20 \%$ at cycle 2 in the IV and IP carboplatin arms. Another $20 \%$ discontinued paclitaxel by cycle 4 and added growth factors (see Data Supplement with regard to dose adjustments). There was no evidence of an increase in Gl perforations, fistulas, or necrosis with IP chemotherapy. Nausea and vomiting grade 3 or worse was more common $(P<.005)$ in the IP cisplatin arm at $11.0 \%$ compared with the IV carboplatin arm at $5.1 \%$ and the IP carboplatin arm at $4.7 \%$. Grade 2 or worse sensory neuropathy was similarly high in all arms, with the IV carboplatin and weekly paclitaxel arm at $30 \%$, and grade 3 or worse sensory neuropathy was similar in all arms at 5.7\%, 4.5\%, and 5.5\%, respectively. Grade 3 or worse hypertension was significantly worse $(P<.005)$ in the IP cisplatin arm at $20.5 \%$ compared with $11.9 \%$ and $14.3 \%$ in the IV carboplatin and 
TABLE 1. Patient Characteristics

\begin{tabular}{|c|c|c|c|c|c|c|c|c|}
\hline \multirow[b]{3}{*}{ Characteristic } & \multicolumn{6}{|c|}{ Randomized Treatment } & & \\
\hline & \multicolumn{2}{|c|}{ IV Carboplatin } & \multicolumn{2}{|c|}{ IP Carboplatin } & \multicolumn{2}{|c|}{ IP Cisplatin } & \multicolumn{2}{|c|}{ Total } \\
\hline & No. & $\%$ & No. & $\%$ & No. & $\%$ & No. & $\%$ \\
\hline \multicolumn{9}{|l|}{ Age-group, years } \\
\hline$<40$ & 27 & 5.2 & 13 & 2.5 & 18 & 3.5 & 58 & 3.7 \\
\hline $40-49$ & 101 & 19.4 & 77 & 14.9 & 95 & 18.2 & 273 & 17.5 \\
\hline $50-59$ & 187 & 35.9 & 178 & 34.4 & 199 & 38.2 & 564 & 36.2 \\
\hline $60-69$ & 152 & 29.2 & 181 & 34.9 & 151 & 29.0 & 484 & 31.0 \\
\hline $70-79$ & 51 & 9.8 & 64 & 12.4 & 53 & 10.2 & 168 & 10.8 \\
\hline$\geq 80$ & 3 & 0.6 & 5 & 1.0 & 5 & 1.0 & 13 & 0.8 \\
\hline \multicolumn{9}{|l|}{ Ethnicity } \\
\hline Hispanic & 17 & 3.3 & 21 & 4.1 & 23 & 4.4 & 61 & 3.9 \\
\hline Non-Hispanic & 469 & 90.0 & 458 & 88.4 & 462 & 88.7 & 1,389 & 89.0 \\
\hline Other/unspecified & 35 & 6.7 & 39 & 7.5 & 36 & 6.9 & 110 & 7.1 \\
\hline \multicolumn{9}{|l|}{ Race } \\
\hline Asian & 15 & 2.9 & 15 & 2.9 & 17 & 3.3 & 47 & 3.0 \\
\hline Black/African American & 17 & 3.3 & 17 & 3.3 & 17 & 3.3 & 51 & 3.3 \\
\hline American Indian/Alaskan Native & 2 & 0.4 & 2 & 0.4 & 2 & 0.4 & 6 & 0.4 \\
\hline Native Hawaiian/Pacific Islander & 1 & 0.2 & 1 & 0.2 & 0 & 0 & 2 & 0.1 \\
\hline White & 473 & 90.8 & 478 & 92.3 & 476 & 91.4 & 1,427 & 91.5 \\
\hline Other/unspecified & 13 & 2.5 & 5 & 1.0 & 9 & 1.7 & 27 & 1.7 \\
\hline \multicolumn{9}{|l|}{ FIGO stage } \\
\hline II & 56 & 10.7 & 56 & 10.8 & 51 & 9.8 & 163 & 10.4 \\
\hline III & 441 & 84.6 & 432 & 83.4 & 432 & 82.9 & 1,305 & 83.7 \\
\hline IV & 24 & 4.6 & 30 & 5.8 & 38 & 7.3 & 92 & 5.9 \\
\hline \multicolumn{9}{|l|}{ Size of residual disease } \\
\hline Microscopic only & 297 & 57.0 & 297 & 57.3 & 306 & 58.7 & 900 & 57.7 \\
\hline $0<$ diameter $\leq 1 \mathrm{~cm}$ & 182 & 34.9 & 189 & 36.5 & 182 & 34.9 & 553 & 35.4 \\
\hline Diameter $>1 \mathrm{~cm}$ & 42 & 8.1 & 32 & 6.2 & 33 & 6.3 & 107 & 6.9 \\
\hline \multicolumn{9}{|l|}{ Histology/grade } \\
\hline Serous/1 & 20 & 3.8 & 12 & 2.3 & 27 & 5.2 & 59 & 3.8 \\
\hline Serous/2 & 43 & 8.3 & 36 & 6.9 & 35 & 6.7 & 114 & 7.3 \\
\hline Serous/3 & 370 & 71.0 & 379 & 73.2 & 377 & 72.4 & 1,126 & 72.2 \\
\hline Endometrioid & 5 & 1.0 & 2 & 0.4 & 4 & 0.8 & 11 & 0.7 \\
\hline Clear cell & 32 & 6.1 & 29 & 5.6 & 26 & 5.0 & 87 & 5.6 \\
\hline Mucinous & 2 & 0.4 & 5 & 1.0 & 5 & 1.0 & 12 & 0.8 \\
\hline Other/not specified & 48 & 9.2 & 55 & 10.6 & 47 & 9.0 & 150 & 9.6 \\
\hline Total & 521 & 33.4 & 518 & 33.2 & 521 & 33.4 & 1,560 & 100.0 \\
\hline
\end{tabular}

Abbreviations: FIGO, International Federation of Gynecology and Obstetrics; IP, intraperitoneal; IV, intravenous.

IP carboplatin arms, respectively. Grade 2 or worse CNS ischemia was seen in the IP cisplatin arm at $2.0 \%$ and led to one death, whereas $0.8 \%$ in the IV carboplatin and $0.6 \%$ in the IP carboplatin arms had at least grade 2 CNS ischemic events reported. Grade 3 or worse thrombotic events, including those that resulted from vascular access devices, occurred in $6.3 \%, 8.4 \%$, and $9.0 \%$, respectively.

\section{PROs}

A total of 1,437 patients (92\%) completed a baseline PRO and at least one follow-up PRO. Completion rates were high 


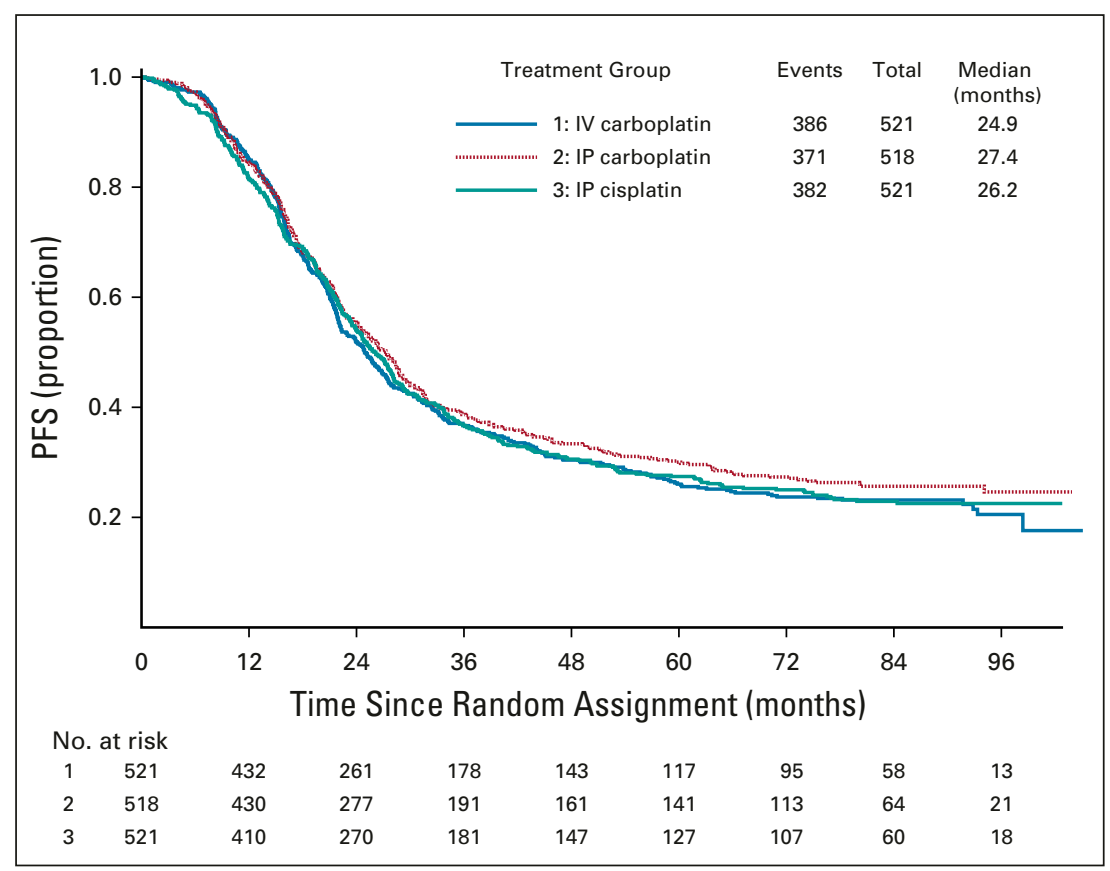

FIG 2. Progression-free survival (PFS) of all randomly assigned participants. In the overall intention-to-treat population of 1,560 patients, 1,139 (73\%) experienced progression. Compared with intravenous (IV) carboplatin, the hazard of first progression or death was $7.5 \%$ lower (hazard ratio, 0.925 ; $95 \% \mathrm{Cl}, 0.802$ to 1.07$)$ for the intraperitoneal (IP) carboplatin arm and $2.3 \%$ lower (hazard ratio, $0.977 ; 95 \% \mathrm{Cl}, 0.847$ to 1.13) for the IP cisplatin arm. There was no statistically significant difference in PFS between the IV regimen and either of the IP regimens.

during active and maintenance therapy, although the overall completion rate was slightly lower in the IP cisplatin arm throughout the study. Before cycle 4, PROs for IP cisplatin were 5.9 points lower $(99 \% \mathrm{Cl}, 3.7$ to 8.1 ; adjusted $P<.001)$ on the basis of the FACT-O TOI compared with those for IV carboplatin and 3.7 points lower $(99 \% \mathrm{Cl}, 1.4$ to 6.0; adjusted $P=.003$ ) than those for IP carboplatin. Although all patients reported increased neurotoxicity during treatment, those in the IP cisplatin arm experienced significantly worse symptoms at cycle 13 compared with IV carboplatin (1.9 points lower; $99 \% \mathrm{Cl}, 1.1$ to 2.6; adjusted $P<.001$ ) and IP carboplatin (1.2 points lower; $99 \% \mathrm{Cl}, 0.4$ to 2.0; adjusted $P=.005)$. Neurotoxicity symptoms did not recover to baseline even 6 months after maintenance therapy among all study arms (Data Supplement). Abdominal discomfort improved across regimens during chemotherapy, but improvement was significantly slower for IP cisplatin compared with IV carboplatin (1.5 points

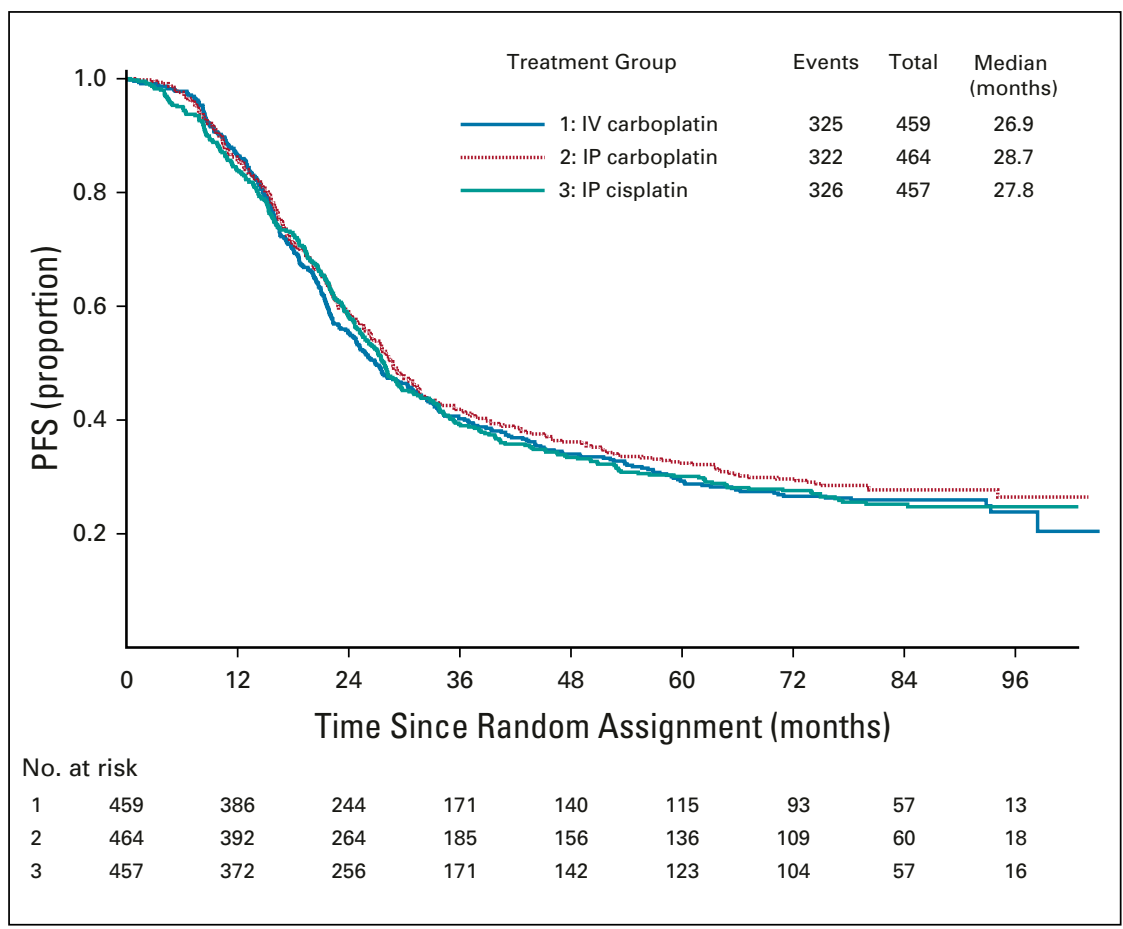

FIG 3. Progression-free survival (PFS) of participants with optimally resected stage II/III with $1 \mathrm{~cm}$ or less residual disease per surgeon. In the 1,380 participants evaluated, $973(71 \%)$ had a median PFS of 26.9 months in the intravenous (IV) carboplatin arm, 28.7 months in the intraperitoneal (IP) carboplatin arm (hazard ratio, $0.921 ; 95 \% \mathrm{Cl}, 0.789$ to 1.07 ), and 27.8 months in the IP cisplatin arm (hazard ratio, $0.966 ; 95 \% \mathrm{Cl}, 0.828$ to 1.13). There was no statistically significant difference in PFS between the IV regimen and either of the IP regimens in this subgroup of patients. 


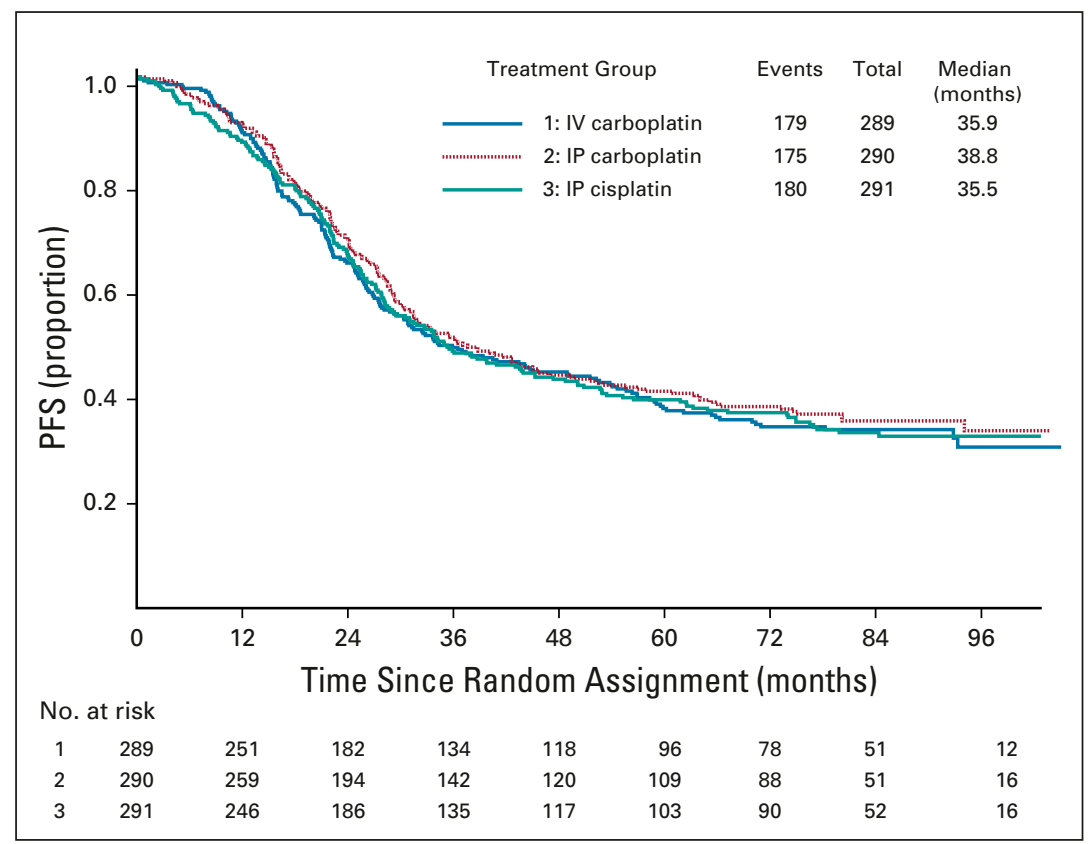

FIG 4. Progression-free survival (PFS) of participants with stage II/III with no gross residual disease. There were 870 participants with stage II or III and no gross residual disease documented at the completion of surgery of whom 534 (61\%) experienced disease progression or death. Adjusted HR $(95 \% \mathrm{Cl})$ of PFS for intraperitoneal (IP) carboplatin vs. intravenous (IV) carboplatin $=0.92(0.75-$ 1.13). Adjusted HR $(95 \% \mathrm{Cl})$ of PFS for IP cisplatin vs. IV carboplatin $=0.97(0.79-$ 1.19). There was no statistically significant difference in PFS between the IV regimen and either of the IP regimens in this subgroup of patients.

lower; 99\% Cl, 1.0 to 2.1; adjusted $P<.001$ ) and IP carboplatin (0.9 points lower; $99 \% \mathrm{Cl}, 0.3$ to 1.5 ; adjusted $P=.005)$. Furthermore, before cycle 7 , patients in the IP cisplatin and IP carboplatin arms reported significantly more abdominal discomfort than those who received the IV regimen ( $P=.006$ and .002 , respectively). The single item "I have nausea" was significantly worse for patients in the IP cisplatin arm compared with IV carboplatin (0.5 points higher; $99 \% \mathrm{Cl}, 0.3$ to 0.7 ; adjusted $P<.001$ ) and IP carboplatin (0.4 points higher; $99 \% \mathrm{Cl}, 0.2$ to 0.6 ; adjusted $P<.001)$. There were no between-arm differences for fatigue in chemotherapy or maintenance therapy PROs.

\section{DISCUSSION}

The primary outcome measure was an improvement in PFS for experimental IP chemotherapy arms compared with the reference arm of every 3 weeks IV carboplatin and IV bevacizumab and weekly paclitaxel. The intention-to-treat analysis shows no statistical difference for PFS among the arms overall or in any subgroup evaluated. Of note, the PFS of patients with optimally resected $1 \mathrm{~cm}$ or less residual stage III disease was 24.9 to 27.1 months (Data Supplement), which is better than that seen in GOG-172, 2,3 where the IV and IP arms showed a PFS of 18.3 and 23.8 months, respectively. In addition, the current study had an OS for

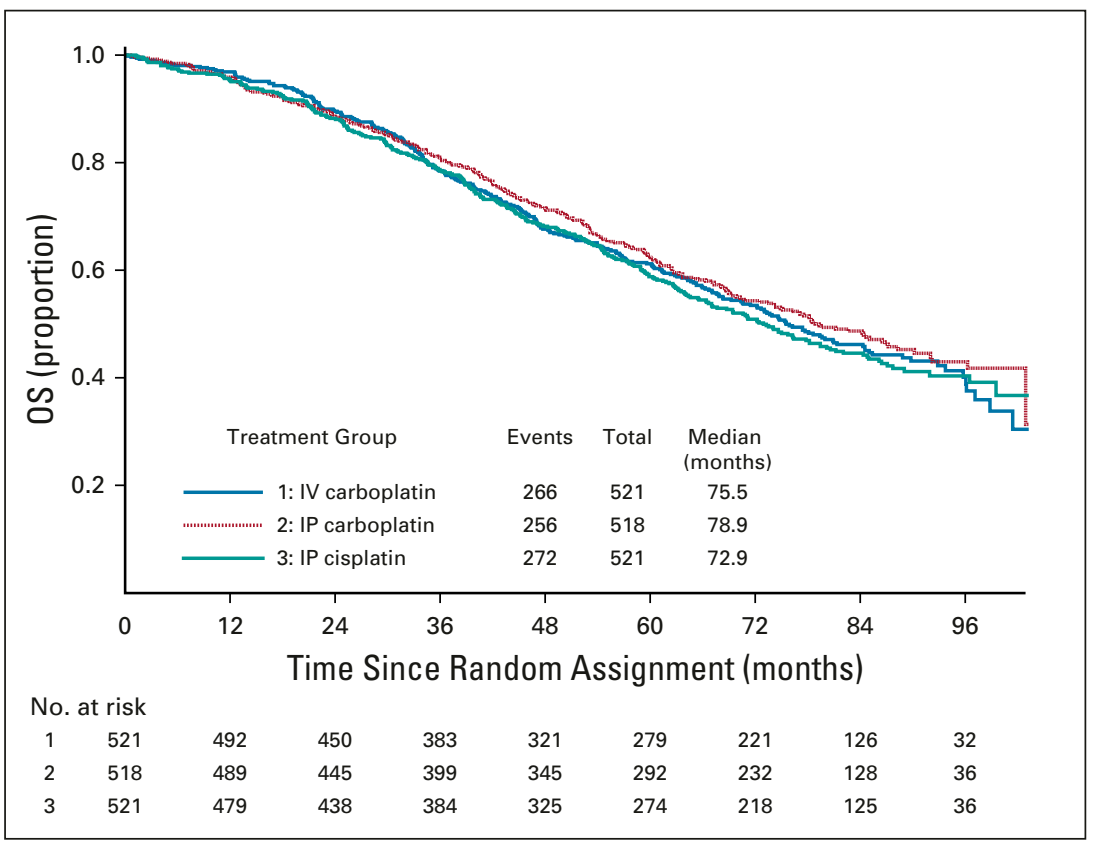

FIG 5. Overall survival (OS) by randomized treatment of all enrolled patients. Forty-nine percent of participants were alive after a median follow-up of 84.8 months. Relative to the intravenous (IV) carboplatin arm, the hazard of death was similar in the intraperitoneal (IP) carboplatin arm (hazard ratio, $0.949 ; 95 \% \mathrm{Cl}, 0.799$ to 1.128 ) and in the IP cisplatin arm (hazard ratio, 1.05; 95\% $\mathrm{Cl}, 0.884$ to 1.24 ). OS for stage II/III with no gross residual disease was 98.8 months for IV carboplatin; 104.8 months for IP carboplatin; and yet to be determined for IP cisplatin, which needs a longer follow-up. 
TABLE 2. Patients Who Experienced Selected Adverse Events by Grade and Randomized Treatment

Grade, \%

\begin{tabular}{|c|c|c|c|c|c|c|c|c|c|c|}
\hline \multirow[b]{3}{*}{ Adverse Event } & & \multirow[b]{3}{*}{$P^{*}$} \\
\hline & \multicolumn{3}{|c|}{ IV Carboplatin $(n=511)$} & \multicolumn{3}{|c|}{ IP Carboplatin $(n=510)$} & \multicolumn{3}{|c|}{ IP Cisplatin $(n=508)$} & \\
\hline & 2 & 3 & $4 / 5$ & 2 & 3 & $4 / 5$ & 2 & 3 & $4 / 5$ & \\
\hline Hemoglobin & 58.5 & 24.7 & 2.0 & 56.7 & 24.1 & 2.2 & 50.4 & 16.1 & 1.8 & $<.001$ \\
\hline Neutrophils & 19.8 & 45.6 & 26.4 & 20.0 & 44.9 & 23.1 & 21.1 & 33.5 & 30.8 & .031 \\
\hline Platelets & 16.8 & 12.9 & 4.7 & 19.0 & 11.6 & 3.5 & 7.3 & 5.5 & 0.6 & $<.001$ \\
\hline Nausea/vomiting & 16.8 & 4.7 & 0.4 & 20.0 & 4.7 & 0 & 25.8 & 10.8 & 0.2 & $<.001$ \\
\hline Infections & 33.7 & 10.2 & 1.4 & 34.3 & 16.1 & 1.2 & 29.6 & 17.0 & 0.8 & .008 \\
\hline GI leak, perforation, or fistula & 1.6 & 2.9 & 2.4 & 1.2 & 3.1 & 0.6 & 1.4 & 3.2 & 1.2 & .492 \\
\hline Thrombus & 2.0 & 2.2 & 4.1 & 1.4 & 4.9 & 3.5 & 1.6 & 4.9 & 4.1 & .206 \\
\hline Hypertension & 17.0 & 11.4 & 0.6 & 15.3 & 13.5 & 0.8 & 17.0 & 18.7 & 1.8 & $<.001$ \\
\hline Sensory neuropathy & 24.1 & 5.5 & 0.2 & 22.6 & 4.5 & 0.0 & 21.3 & 5.5 & 0.0 & .663 \\
\hline CNS ischemia & 0.4 & 0.4 & 0.0 & 0.0 & 0.2 & 0.4 & 0.2 & 1.0 & 0.8 & .058 \\
\hline
\end{tabular}

NOTE. This summary of adverse events does not include 31 patients who did not initiate their assigned study treatment.

Abbreviations: IP, intraperitoneal; IV, intravenous.

*Nominal $P$ value for a test of the null hypothesis that the probability of a grade 3 or worse adverse event is equal over the three treatments.

stage III optimally resected to $1 \mathrm{~cm}$ or less of 74.1 to 78.2 months (Data Supplement), which compares favorably to that of GOG-172, where survival was 49.7 and 65.6 months in the IV and IP arms, respectively. In the current study, $57 \%$ of the patients with stage II/II disease were classified as having RO (no residual disease), and their OS was 98.8 months for IV carboplatin, 104.8 months for IP carboplatin, and not reached for IP cisplatin (Data Supplement). This compares favorably with an ancillary analysis of patients with no gross residual disease in GOG-114 and GOG-172 treated with IP chemotherapy, where the median OS was 110 months. ${ }^{2}$ This comparison brings use of bevacizumab maintenance into question for this particular population, although it must be acknowledged that this observation is based on cross-study comparisons. It should be noted that the IP carboplatin arm was very well tolerated (Table 2) and that only grade 3 infections and abdominal pain were worse than in the IV reference arm.

There were 178 patients with surgically resected suboptimal stage III and IV disease enrolled as an exploratory objective, and the results did not show a benefit of IP chemotherapy. The IV carboplatin arm demonstrated a PFS of 16.9 months and OS of 55.5 months (Data Supplement), which compares favorably with the GOG- $218^{15}$ results of a PFS of 14 months and OS of 39 months and the GOG$262^{11}$ PFS of 14.7 and OS of 40.2 months. The explanation of these improved results could be secondary to the required primary surgical resection.

On June 13, 2018, the Food and Drug Administration approved the administration of bevacizumab in combination with carboplatin and paclitaxel on the basis of GOG$218 .{ }^{15}$ The current study included bevacizumab therapy, and participants had excellent PFS and OS in all subgroups enrolled. The administration of paclitaxel once per week at $80 \mathrm{mg} / \mathrm{m}^{2}$ versus every 3 weeks paclitaxel at $175 \mathrm{mg} / \mathrm{m}^{2}$ has conflicting data. The JGOG study favored weekly dosing, ${ }^{16}$ whereas the ICON-8 Gynecologic Cancer Intergroup study reported by Clamp et $\mathrm{al}^{25}$ at the European Society of Medical Oncology meeting did not demonstrate a benefit of weekly paclitaxel administration compared with every 3 weeks. The secondary analysis of GOG-262 by Chan et $\mathrm{al}^{11}$ suggests that when bevacizumab is added, then weekly paclitaxel shows no advantage compared with the use of conventional every-3-week dosing. The administration of all chemotherapy every 3 weeks is more convenient and has less sensory neuropathy. ${ }^{11}$

The modification of the cisplatin dose from $100 \mathrm{mg} / \mathrm{m}^{2}$ down to $75 \mathrm{mg} / \mathrm{m}^{2}$ and the reduction in paclitaxel dose to $135 \mathrm{mg} / \mathrm{m}^{2}$ over 3 hours instead of 24 hours to decrease toxicity and allow more tolerable outpatient administration may have compromised the efficacy of the IP cisplatin arm overall. In addition, the interaction between cisplatin and bevacizumab increased hypertension, and more participants (30\%) discontinued bevacizumab and received fewer cycles of chemotherapy (84\%) in the IP cisplatin arm because of this toxicity. Dropping bevacizumab may improve tolerance of the modified IP cisplatin arm and allow more patients to complete the recommended cycles, unlike in GOG-172 where only $42 \%$ of patients received six cycles of IP cisplatin. Nonetheless, data from the current study suggest no advantage to the use of the modified IP cisplatin regimen compared with the more conventional IV drug administration.

All arms of the current study compare favorably with hyperthermic IP chemotherapy where the PFS was 14.2 months and OS 33.9 months for patients with stage III 
resected to $1 \mathrm{~cm}$ or less disease. ${ }^{26}$ In addition, Intraperitoneal Therapy for Ovarian Cancer With Carboplatin (iPocc), a study conducted by the Gynecologic Cancer Intergroup and JGOG that will be reported in 2020, is comparing weekly IV paclitaxel with IP versus IV carboplatin without bevacizumab. The results of iPocc should provide additional insight into the role of IP therapy in the context of weekly IV paclitaxel and may clarify the confounding role of bevacizumab in interpreting the data from GOG-252. In that regard, it is possible that the addition of bevacizumab interacts with chemotherapy, which makes any differences between arms not evident.

The optimal maintenance strategy in the first-line setting is under active investigation. Genomic mutation analysis has not yet been performed on the participants of GOG-252, but this will be an important area of future research, especially as it relates to the use of inhibitors of poly (ADP-ribose) polymerase (PARP). In that regard, the PARP inhibitor olaparib is now preferred over maintenance bevacizumab in genomic BRCA1 and BRCA2 mutation carriers and for tumors that harbor somatic BRCA1 or BRCA2 mutations. ${ }^{27}$ Concurrent administration of bevacizumab and PARP inhibitors as maintenance therapy is under investigation in the PAOLA-1 study, which is set to report in 2019

\author{
AFFILIATIONS \\ ${ }^{1}$ University of Oklahoma Health Sciences Center, Oklahoma City, OK \\ ${ }^{2}$ Roswell Park Cancer Institute, Buffalo, NY \\ ${ }^{3}$ University of California, Irvine, Medical Center, Orange, CA \\ ${ }^{4}$ The University of Chicago Medicine, Chicago, IL \\ ${ }^{5}$ Women and Infants Hospital, Providence, RI \\ ${ }^{6}$ Saitama Medical University International Medical Center, Hidaka-Shi, \\ Japan \\ ${ }^{7}$ The University of Arizona Cancer Center, Tucson, AZ \\ ${ }^{8}$ The University of Arizona, Tucson, AZ \\ ${ }^{9}$ The Ohio State University, Columbus, $\mathrm{OH}$ \\ ${ }^{10}$ Washington University in St Louis, St Louis, MO \\ ${ }^{11}$ University of North Carolina at Chapel Hill, Chapel Hill, NC \\ ${ }^{12}$ University of Colorado, Aurora, CO \\ ${ }^{13}$ University of Washington School of Medicine, Seattle, WA \\ ${ }^{14}$ Cleveland Clinic Foundation, Cleveland, $\mathrm{OH}$ \\ ${ }^{15}$ Memorial Sloan Kettering Cancer Center, New York, NY \\ ${ }^{16}$ University of Connecticut, Storrs, CT \\ ${ }^{17}$ Duke University Health System, Durham, NC \\ ${ }^{18}$ University of Pennsylvania, Philadelphia, PA
}

\section{CORRESPONDING AUTHOR}

Joan L. Walker, MD, Stephenson Cancer Center, University of Oklahoma Health Sciences Center, 800 NE 10th St, Oklahoma City, OK 73104; e-mail: joan-walker@ouhsc.edu.

\section{PRIOR PRESENTATION}

Presented at the Society of Gynecologic Oncology Annual Meeting, March 12, 2016; American Society of Clinical Oncology 2016 Annual Meeting, Chicago, IL, June 3-7, 2016; and NRG Oncology, Houston, TX, February 9-11, 2017.

\section{SUPPORT}

Supported by National Cancer Institute (NCl) grants to NRG Oncology (U10 CA180822) and NRG Operations (U10 CA180868) as well as by
(ClinicalTrials.gov identifier: NCT02477644). Homologous repair deficiency testing on tumors is another potential tool to select treatment with PARP inhibitors. Interest also exists in using IP cisplatin in these patients because of their DNA repair deficiency and excellent survival outcomes with highdose cisplatin delivered through the IP route. In particular, Lesnock et $\mathrm{al}^{28}$ determined in a secondary analysis of GOG172 that aberrant BRCA1 expression improves survival from 47 months when treated with IV cisplatin to 84 months when treated with IP cisplatin. Translational studies using GOG-252 specimens may identify subsets of patients who may benefit from IP therapy.

In conclusion, the benefit of IP administration of chemotherapy was not evident in this large randomized clinical trial. The toxicity and PROs support the decision to select the IV carboplatin AUC 6 day 1, dose-dense paclitaxel $80 \mathrm{mg} / \mathrm{m}^{2} \mathrm{IV}$ once per week, and bevacizumab $15 \mathrm{mg} / \mathrm{kg}$ IV day 1 given in 3-week cycles, and there is no evidence that this compromises PFS or OS. The use of IP chemotherapy may still be an option in select patients who are homologous repair deficiency positive with no gross residual disease, but the original GOG-172 dose and schedule should be considered in that case and may be used without bevacizumab.

$\mathrm{NCl}$ Community Oncology Research Program grant UG1 CA189867. The NCl-supplied agent was bevacizumab (NSC \#704865, IND \#7921). GOG-0252 was conducted under a cooperative research and development agreement between the $\mathrm{NCl}$ Cancer Therapy Education Program and Genentech. No additional funding support was provided to the Gynecologic Oncology Group. Bevacizumab was distributed by the Pharmaceutical Management Branch at $\mathrm{NCl}$.

AUTHORS' DISCLOSURES OF POTENTIAL CONFLICTS OF INTEREST AND DATA AVAILABILITY STATEMENT

Disclosures provided by the authors and data availability statement (if applicable) are available with this article at DOI https://doi.org/10.1200/ JC0.18.01568.

\section{AUTHOR CONTRIBUTIONS}

Conception and design: Joan L. Walker, Mark F. Brady, Lari Wenzel, Gini F. Fleming, Paul A. DiSilvestro, Keiichi Fujiwara, David S. Alberts, Peter G. Rose, Carol Aghajanian, Robert S. Mannel

Administrative support: Matthew A. Powell, Angeles Alvarez Secord Provision of study material or patients: Joan L. Walker, Paul A. DiSilvestro, Krishnansu S. Tewari, David E. Cohn, Matthew A. Powell, Susan A. Davidson, Heidi J. Gray, Peter G. Rose, Tashanna Myers, Angeles Alvarez Secord, Stephen C. Rubin

Collection and assembly of data: Joan L. Walker, Gini F Fleming, Helen Q. Huang, Paul A. DiSilvestro, Wenxin Zheng, Krishnansu S. Tewari, Matthew A. Powell, Peter G. Rose, Tashanna Myers, Angeles Alvarez Secord

Data analysis and interpretation: Joan L. Walker, Mark F. Brady, Lari Wenzel, Helen Q. Huang, Paul A. DiSilvestro, Keiichi Fujiwara, David E. Cohn, Matthew A. Powell, Linda Van Le, Susan A. Davidson, Peter G. Rose, Tashanna Myers, Angeles Alvarez Secord, Robert S. Mannel Manuscript writing: All authors

Final approval of manuscript: All authors

Accountable for all aspects of the work: All authors 
ACKNOWLEDGMENT

The following GOG member institutions participated in the primary treatment studies: Cancer Trials Support Unit; University of Oklahoma Health Sciences Center; University of California Medical Center Irvine-Orange Campus; The Ohio State University Comprehensive Cancer Center; Women and Infants Hospital; University of North Carolina at Chapel Hill; Washington University School of Medicine; Abramson Cancer Center of the University of Pennsylvania; University of Colorado Cancer Center-Anschutz Cancer Pavilion; Fred Hutchinson Cancer Research Center; Cleveland Clinic Foundation; Memorial Sloan Kettering Cancer Center; The Hospital of Central Connecticut; Duke University Medical Center; Walter Reed National Military Medical Center; Roswell Park Cancer Institute; University of Alabama at Birmingham; Stony Brook University Medical Center; University of Minnesota Medical

Center-Fairview; University of Wisconsin Hospital and Clinics; University of lowa Hospitals and Clinics; Mayo Clinic; Moffitt Cancer Center and Research Institute; University of Kentucky; Cooper Hospital University Medical Center; University of Pittsburgh Cancer Institute; Northwestern University; Rush University Medical Center; Cancer Research Consortium of West Michigan NCl Community Oncology Research Program (NCORP); Women's Cancer Center of Nevada; Fox Chase Cancer Center; MetroMinnesota Community Clinical Oncology Program (CCOP); University of New Mexico; Case Western Reserve University; Aurora Women's Pavilion of Aurora West Allis Medical Center; University of Virginia; University of California, San Francisco, Mount Zion; Cancer Research for the Ozarks NCORP; University of Mississippi Medical Center; Greenville Health System Cancer Institute/Greenville CCOP; Abington Memorial Hospital; University of Texas Southwestern Medical Center; Indiana University Hospital/Melvin and Bren Simon Cancer Center; Gynecologic Oncology of West Michigan; lowa-Wide Oncology Research Coalition NCORP; Penn State Milton S. Hershey Medical Center; MD Anderson Cancer Center; University of Cincinnati; Wake Forest University Health Sciences; University of Massachusetts Memorial Health Care; University of Chicago; Wayne State University/ Karmanos Cancer Institute; Virginia Commonwealth University; Yale University; University of California, Los Angeles, Health System; Georgia Center for Oncology Research and Education; Evanston CCOP-NorthShore University HealthSystem; Michigan Cancer Research Consortium CCOP; Mount Sinai School of Medicine; St Joseph Hospital and Medical Center; University of Hawaii; Kalamazoo CCOP; Delaware/Christiana Care CCOP; Carolinas Medical Center/ Levine Cancer Institute; Central Illinois CCOP; Virginia Mason CCOP; Queens Hospital Center; University of Texas Medical Branch at Galveston; Wisconsin NCORP; Northern Indiana Cancer Research Consortium; Scott and White Memorial Hospital; St Vincent Hospital; and Wichita CCOP.

\section{REFERENCES}

1. Torre LA, Trabert B, DeSantis CE, et al: Ovarian cancer statistics, 2018. CA Cancer J Clin 68:257-296, 2018

2. Landrum LM, Java J, Mathews CA, et al: Prognostic factors for stage III epithelial ovarian cancer treated with intraperitoneal chemotherapy: A Gynecologic Oncology Group study. Gynecol Oncol 130:12-18, 2013

3. Armstrong DK, Bundy B, Wenzel L, et al: Intraperitoneal cisplatin and paclitaxel in ovarian cancer. N Engl J Med 354:34-43, 2006

4. Landrum LM, Gold MA, Moore KN, et al: Intraperitoneal chemotherapy for patients with advanced epithelial ovarian cancer: A review of complications and completion rates. Gynecol Oncol 108:342-347, 2008

5. Wright AA, Cronin A, Milne DE, et al: Use and effectiveness of intraperitoneal chemotherapy for treatment of ovarian cancer. J Clin Oncol 33:2841-2847, 2015

6. Dizon DS, Sill MW, Gould N, et al: Phase I feasibility study of intraperitoneal cisplatin and intravenous paclitaxel followed by intraperitoneal paclitaxel in untreated ovarian, fallopian tube, and primary peritoneal carcinoma: A Gynecologic Oncology Group study. Gynecol Oncol 123:182-186, 2011

7. Gould N, Sill MW, Mannel RS, et al: A phase I study with an expanded cohort to assess feasibility of intravenous docetaxel, intraperitoneal carboplatin and intraperitoneal paclitaxel in patients with previously untreated ovarian, fallopian tube or primary peritoneal carcinoma: A Gynecologic Oncology Group study. Gynecol Oncol 127:506-510, 2012

8. Morgan MA, Sill MW, Fujiwara K, et al: A phase I study with an expanded cohort to assess the feasibility of intraperitoneal carboplatin and intravenous paclitaxel in untreated ovarian, fallopian tube, and primary peritoneal carcinoma: A Gynecologic Oncology Group study. Gynecol Oncol 121:264-268, 2011

9. Katsumata N, Yasuda M, Isonishi S, et al: Long-term results of dose-dense paclitaxel and carboplatin versus conventional paclitaxel and carboplatin for treatment of advanced epithelial ovarian, fallopian tube, or primary peritoneal cancer (JGOG 3016): A randomised, controlled, open-label trial. Lancet Oncol 14: 1020-1026, 2013

10. Markman M, Blessing J, Rubin SC, et al: Phase II trial of weekly paclitaxel $\left(80 \mathrm{mg} / \mathrm{m}^{2}\right)$ in platinum and paclitaxel-resistant ovarian and primary peritoneal cancers: A Gynecologic Oncology Group study. Gynecol Oncol 101:436-440, 2006

11. Chan JK, Brady MF, Penson RT, et al: Weekly vs. every-3-week paclitaxel and carboplatin for ovarian cancer. N Engl J Med 374:738-748, 2016

12. Arora N, Tewari D, Cowan C, et al: Bevacizumab demonstrates activity in advanced refractory fallopian tube carcinoma. Int J Gynecol Cancer 18:369-372, 2008

13. Cannistra SA, Matulonis UA, Penson RT, et al: Phase II study of bevacizumab in patients with platinum-resistant ovarian cancer or peritoneal serous cancer. J Clin Oncol 25:5180-5186, 2007

14. Burger RA, Sill MW, Monk BJ, et al: Phase II trial of bevacizumab in persistent or recurrent epithelial ovarian cancer or primary peritoneal cancer: A Gynecologic Oncology Group study. J Clin Oncol 25:5165-5171, 2007

15. Burger RA, Brady MF, Bookman MA, et al: Incorporation of bevacizumab in the primary treatment of ovarian cancer. N Engl J Med 365:2473-2483, 2011

16. Oza AM, Cook AD, Pfisterer J, et al: Standard chemotherapy with or without bevacizumab for women with newly diagnosed ovarian cancer (ICON7): Overall survival results of a phase 3 randomised trial. Lancet Oncol 16:928-936, 2015

17. Basen-Engquist K, Bodurka-Bevers D, Fitzgerald MA, et al: Reliability and validity of the functional assessment of cancer therapy-ovarian. J Clin Oncol 19: 1809-1817, 2001

18. Yost KJ, Eton DT: Combining distribution- and anchor-based approaches to determine minimally important differences: The FACIT experience. Eval Health Prof 28:172-191, 2005

19. Pocock SJ, Simon R: Sequential treatment assignment with balancing for prognostic factors in the controlled clinical trial. Biometrics 31:103-115, 1975

20. Eisenhauer EA, Therasse P, Bogaerts J, et al: New response evaluation criteria in solid tumours: Revised RECIST guideline (version 1.1). Eur J Cancer 45: 228-247, 2009

21. Rustin GJS, Marples M, Nelstrop AE, et al: Use of CA-125 to define progression of ovarian cancer in patients with persistently elevated levels. J Clin Oncol 19: 4054-4057, 2001

22. Mantel N: Evaluation of survival data and two new rank order statistics arising in its consideration. Cancer Chemother Rep 50:163-170, 1966

23. Cancer Therapy Evaluation Program: Common Terminology Criteria for Adverse Events v3.0 (CTCAE), 2006. https://ctep.cancer.gov/protocoldevelopment/ electronic_applications/docs/ctcaev3.pdf 
24. Hochberg Y: A sharper Bonferroni procedure for multiple tests of significance. Biometrika 75:800-802, 1988

25. Clamp AR, McNeish I, Dean A, et al: ICON8: A GCIG phase III randomised trial evaluating weekly dose-dense chemotherapy integration in first-line epithelial ovarian/fallopian tube/primary peritoneal carcinoma (EOC) treatment: Results of primary progression-free survival (PFS) analysis. Ann Oncol 28, 2017 (suppl 5, abstr 9290_PR)

26. van Driel WJ, Koole SN, Sonke GS: Hyperthermic intraperitoneal chemotherapy in ovarian cancer. N Engl J Med 378:230-240, 2018

27. Moore K, Colombo N, Scambia G, et al: Maintenance olaparib in patients with newly diagnosed advanced ovarian cancer. N Engl J Med 379:2495-2505, 2018

28. Lesnock JL, Darcy KM, Tian C, et al: BRCA1 expression and improved survival in ovarian cancer patients treated with intraperitoneal cisplatin and paclitaxel: A Gynecologic Oncology Group study. Br J Cancer 108:1231-1237, 2013

\section{ASCOconnection.org: The Professional Networking Site That Keeps You Connected With Oncology Care Providers Across the Globe}

- Expand your online network and share your experiences

- Read personal reflections from engaging practitioners

- Stay current with updates from ASCO leadership

- Write your own blog

- View career advice for trainee and early-career oncologists

- Read articles from your member magazine

- Start your own discussion on any oncology topic

Contact ascoconnection@asco.org to contribute.

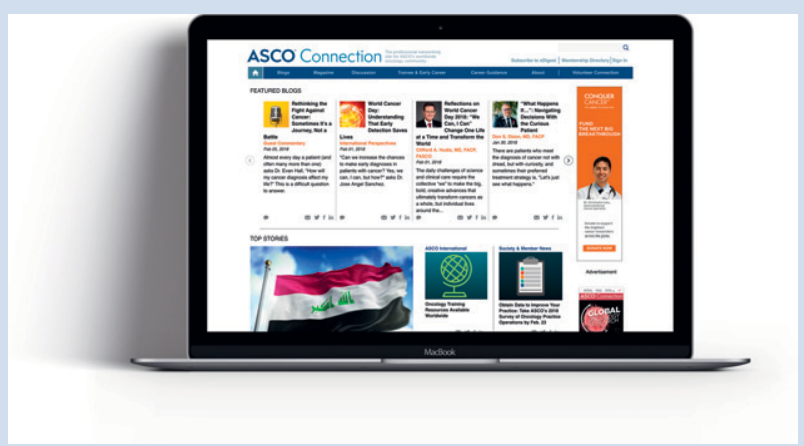

ASCO 
Randomized Trial of Intravenous Versus Intraperitoneal Chemotherapy Plus Bevacizumab in Advanced Ovarian Carcinoma: An NRG Oncology/Gynecologic Oncology Group Study

The following represents disclosure information provided by authors of this manuscript. All relationships are considered compensated. Relationships are self-held unless noted. I = Immediate Family Member, Inst = My Institution. Relationships may not relate to the subject matter of this manuscript. For more information about ASCO's conflict of interest policy, please refer to www.asco.org/rwc or ascopubs.org/jco/site/ifc.

Joan L. Walker

Research Funding: US Biotest, Genentech

Mark F. Brady

Consulting or Advisory Role: Cel-Sci

\section{Gini F. Fleming}

Research Funding: Corcept Therapeutics (Inst), AbbVie (Inst), Genentech (Inst) Tesaro (Inst), Syndax (Inst), Forty Seven (Inst), lovance Biotherapeutics (Inst), Syros Pharmaceuticals (Inst), Astex Pharmaceuticals (Inst), Merck (Inst)

\section{Paul A. DiSilvestro}

Consulting or Advisory Role: AstraZeneca, Tesaro

Research Funding: Janssen Pharmaceuticals (Inst), Tesaro (Inst), AstraZeneca (Inst), Genentech (Inst), AbbVie (Inst), Johnson \& Johnson (Inst)

\section{Keiichi Fujiwara}

Honoraria: Chugai Pharmaceutical, Kyowa Hakko Kirin, Zeria Pharmaceutical, Nippon Kayaku, Taiho Pharmaceutical, Daiichi Sankyo, Ono Pharmaceutical, Bayer AG

Consulting or Advisory Role: MSD, Eisai, Pfizer

Research Funding: Eisai (Inst), Taiho Pharmaceutical (Inst), Pfizer (Inst), Kaken Pharmaceutical (Inst), Immunogen (Inst), Ono Pharmaceutical (Inst),

AstraZeneca (Inst), Oncotherapeutics (Inst), Zeria Pharmaceutical (Inst), MSD (Inst)

Travel, Accommodations, Expenses: Pfizer

\section{Krishnansu S. Tewari}

Honoraria: Tesaro, Clovis Oncology

Consulting or Advisory Role: Roche, Genentech

Speakers' Bureau: Roche, Genentech, AstraZeneca, Merck

Research Funding: AbbVie (Inst), Genentech (Inst), Roche (Inst), Morphotek (Inst), Merck (Inst), Regeneron Pharmaceuticals (Inst)

Travel, Accommodations, Expenses: Roche, Genentech

\section{David E. Cohn}

Consulting or Advisory Role: Oncology Analytics

Research Funding: NRG Oncology (Inst), Advaxis (Inst), Agenus (Inst), Ajinomoto (Inst), Array BioPharma (Inst), AstraZeneca (Inst), Bristol-Myers Squibb (Inst), Clovis Oncology (Inst), ERGOMED Clinical Research (Inst), Exelixis (Inst), Genentech (Inst), GlaxoSmithKline (Inst), Gynecologic Oncology Group (Inst), ImmunoGen (Inst), INC Research (Inst), inVentiv Health Clinical (Inst), Janssen Pharmaceuticals (Inst), Ludwig Institute for Cancer Research (Inst), PRA International (Inst), EMD Serono (Inst), Stemcentrx (Inst), Tesaro (Inst), AbbVie (Inst), Henry Jackson Foundation (Inst), PharmaMar (Inst), Sanofi (Inst), Eisai (Inst), Pfizer (Inst), Novartis (Inst), Regeneron Pharmaceuticals (Inst),

Tricon Pharmaceuticals (Inst)

Other Relationship: Elsevier, UpToDate

\section{Matthew A. Powell}

Consulting or Advisory Role: Roche, Genentech, AstraZeneca, Tesaro, Clovis Oncology

Speakers' Bureau: Genentech, Roche, AstraZeneca, Tesaro, Clovis Oncology

\section{Linda Van Le}

Consulting or Advisory Role: Eye Point (I), Novartis (I), Eyevensys (I), Bayer AG (I)

Research Funding: Gynecologic Oncology Group Partners Trial (Inst)

\section{Carol Aghajanian}

Consulting or Advisory Role: Clovis Oncology, Tesaro, Mateon Therapeutics, Immunogen

Research Funding: Genentech (Inst), Roche (Inst), AbbVie (Inst), Clovis Oncology (Inst), AstraZeneca (Inst)

\section{Angeles Alvarez Secord}

Honoraria: Genentech, Roche, Janssen Pharmaceuticals, AstraZeneca, Clovis Oncology, Astex Pharmaceuticals, Tesaro, Alexion Pharmaceuticals, Myriad Pharmaceuticals, Arivave, Mersana, Oncoquest, Merck

Consulting or Advisory Role: Genentech, Roche, Janssen Pharmaceuticals, AstraZeneca, Clovis Oncology, Tesaro, Astex Pharmaceuticals, Alexion Pharmaceuticals, Myriad Genetics, Arivave, Mersana, Oncoquest, Merck Research Funding: Tesaro (Inst), AstraZeneca (Inst), Amgen (Inst), Genentech (Inst), Boehringer Ingelheim (Inst), Prima BioMed (Inst), AbbVie (Inst), Merck (Inst), PharmaMar (Inst), Taplmmune (Inst)

Robert S. Mannel

Consulting or Advisory Role: Clovis Oncology (Inst), Tesaro (Inst)

No other potential conflicts of interest were reported. 